\title{
In der Moralfalle?
}

\section{Eine systemtheoretisch inspirierte Reflexion ethischer Politikberatung}

\author{
Axel Bohmeyer
}

\begin{abstract}
Kernaussagen
Manövrieren sich Politiker, die eine moralgeladene Kommunikation verwenden, in eine ethisch problematische Moralfalle? Und kann eine ethische Politikberatung, die von Politikern bei moralisch strittigen Fragen in Anspruch genommen wird, einen Ausweg weisen? . Der Ratschlag des Systemtheoretikers Luhmann besagt, dass die Aufgabe der Ethik darin liegen könnte, dem politischen System gute Gründe dafür zu liefern, wann moralische Kommunikation verwendet werden sollte und wann nicht. In diesem Beitrag werden die systemtheoretischen Überlegungen zur Unterscheidung von Moral und Ethik skizziert, die mit Blick auf die ethische Reflexion ethischer Politikberatung als kritisches Element in Stellung gebracht werden sollen. Es wird das Gefährdungspotenzial moralischer Kommunikation analysiert, das sich im Rahmen der Debatte über die Anwendung der Präimplantationsdiagnostik beobachten lässt. Abschließend wird mit dem „irrenden Gewissen“" (Thomas von Aquin) ein komplexer ethischer Theorieansatz diskutiert, der aus der Moralfalle herausführen könnte.
\end{abstract}

Moralisten ,sind nur in kleinen Zablen erträglich, nur unter der Voraussetzung, dass von niemandem sonst ein derart heroischen Verhalten verlangt wird, und außerdem nur dann, wenn ibnen der Zugang zu den Guillotinen verweigert wird. " (Lubmann, 2008 a, S. 183)

\section{Einleitung}

Am 17. Februar 2012 trat Christian Wulff von seinem Amt als Bundespräsident der Bundesrepublik Deutschland zurück. Dem Rücktritt ging eine lange öffentlichen Diskussion voraus, die sich an den zögerlichen und widersprüchlichen Angaben Christian Wulffs zu einem umstrittenen Privatkredit entzündete. Im Rahmen der Berichterstattung listete die Frankfurter Allgemeine Zeitung auf der zweiten Seite ihrer Ausgabe vom 21. Dezember 2011 - ohne weitere Kommentierung seitens der Redaktion - eine Auswahl einschlägiger öffentlicher Äußerungen Wulffs auf, die dieser vor seiner Zeit als Bundespräsident gemacht hatte. Darunter findet sich folgende Aussage des damaligen stellvertretenden Vorsitzenden der CDU: „Ich leide physisch darunter, dass wir keinen unbefangenen Bundespräsidenten haben." So wurde Wulff am 31. Januar 2000 im Rahmen eines Artikels des Nachrichtenmagazins FOCUS wiedergegeben, in dem über die „Düsseldorfer Flugaffäre“ berichtet wurde, in die der damalige Bundespräsidenten Johannes Rau involviert war. ${ }^{1}$ Dieser hatte zuvor zugegeben, in seiner
Zeit als Ministerpräsident des Landes Nordrhein-Westfalen von der Westdeutschen Landesbank finanzierte Flüge genutzt zu haben, um Termine der SPD wahrzunehmen.

Die „Affäre Wulff“ hätte der Soziologe Niklas Luhmann umstandslos in seine systemtheoretische, und das heißt immer auch differenzorientierte, Reflexion der Moral einbinden können. „Wer moralisch kommuniziert und sich als Autor kenntlich macht, muss akzeptieren, dass seine Kriterien auch auf ihn selbst angewandt werden. Das ist eines der Probleme, das sich bei der Entdeckung und Ausnutzung politischer Skandale ergibt [...]. Die moralische Kommunikation wirkt also auf den zurück, der sie riskiert.“ (Luhmann, 2008 a, S. 188) Deshalb sollte der Autor diese mögliche Rückbindung und die damit verbundenen möglichen Folgen im Vorfeld seiner Kommunikation bedenken (vgl. dazu Luhmann, 2008 c, S. 278). Die Rückwirkung der riskanten moralischen Kommunikation auf den Autor Wulff gipfelte letztlich in der Überschrift eines Leitartikels der Berliner Zeitung vom 2. Januar 2012: „Moralisch gescheitert" (Hebel, 2012, S.4) war hier mit Blick auf den Bundespräsidenten zu lesen. ${ }^{2}$ Am 4. Januar 2012 machte Wulff diese Rückbindung der moralischen Kommunikation dann selbst zum Thema. Im Fernsehinterview mit Bettina Schausten (ZDF) und Ulrich Deppendorf (ARD) machte er deutlich, dass sein eigener moralischer Geltungsanspruch auf ihn zurückgefallen war. Konfrontiert mit seinen oben genannten Aussagen aus dem Jahr 2000 äußerte sich Wulff wie folgt: „Also wir müssen alle hohe Ansprüche haben in dem Wissen, dass wir alle fehlbar sind. Und natürlich denkt man viel jetzt über die Bibelstelle nach: Derjenige, der ohne Schuld ist, werfe den ersten Stein. Und alle gingen bei dieser Steinigung. Weil allen klar wurde: Also Vorsicht, wenn Du mit einem Finger auf andere zeigst, zeigen andere auf Dich selbst. Insofern wird

1 In der am 30. Januar 2000 erschienenen Berliner Tageszeitung B.Z. äußerte sich Wulff in einem Interview wie folgt: „Es ist tragisch, dass Deutschland in dieser schwierigen Zeit keinen unbefangenen Bundespräsidenten hat, der seine Stimme mit Autorität erheben kann. Es handelt sich in NRW offensichtlich um eine Verfilzung mit schwarzen Reise-Kassen jenseits der parlamentarischen Kontrolle. Dies stellt eine Belastung des Amtes und für Johannes Rau dar."

2 Riskant war Christian Wulffs moralische Kommunikation nicht nur angesichts seiner Äußerungen zu seinem damaligen Vorgänger im Amt Johannes Rau, sondern auch mit Blick auf die Veröffentlichung eines Interview-Buches: „Besser die Wahrheit“ lautet der Titel des Buches (vgl. Müller-Vogg \& Wulff, 2007). Zudem zeigt sich: Die moralgeladene Kommunikation wird für den Politiker noch riskanter, wenn er sie mit Hilfe der Massenmedien verbreitet. Zu leicht macht es sich allerdings derjenige, der die moralgeladene Kommunikation des zurückgetretenen Bundespräsidenten Wulff einfach als moralisch zu verurteilende Heuchelei auszuweist. Zumindest für Luhmann würden mit einem solchen moralischen Urteil die Möglichkeiten und Aufgaben einer ethischen Reflexion unterschritten. 
man auch lebensklüger. Und heute kann ich Johannes Rau besser verstehen, als ich ihn damals verstanden habe.”

Wenn sich die Verwendung einer moralgeladenen Kommunikation schon im politischen Alltagsgeschäft für den Politiker als derart risikoreich erweist und mit Missbilligung der Person bestraft wird, wie gefährlich muss sie dann erst sein, wenn sie im Kontext eigens ausgewiesener, kontroverser moralischer Fragestellungen verwendet wird? Welche Risiken entstehen, wenn die Volksvertreter dezidiert strittige Moralfragen diskutieren? Und kann eine ethische Politikberatung ihnen bei der Diskussion hilfreich zur Seite stehen? Wie geht das politische System mit solchen Personen um, die vorgebrachte und mehrheitlich geteilte moralische Positionen nicht teilen und sich im weiteren Verlauf der Debatten auch nicht durch eine moralische Kommunikation überzeugen lassen? Wenn diese Personen im Verlauf einer Diskussion einer moralisch strittigen Position moralisch disqualifiziert werden, dann stellt sich die Frage, ob man mit ihnen mit Blick auf andere politisch relevante Fragen in demokratisch verfassten Abstimmungsprozessen noch Allianzen schließen darf. Manövrieren sich Politiker, die eine moralgeladene Kommunikation verwenden, also in eine ethisch problematische Moralfalle und kann eine ethische Politikberatung einen Ausweg aus einer solchen Moralfalle weisen?

Die folgenden Ausführungen vertiefen die systemtheoretischen Reflexionen Niklas Luhmanns zur moralischen Kommunikation im politischen System und nutzen seine für Ethiker - die sich bewusst auf die Identität ihres Fachs berufen und professionell betriebene Ethik als eine eigenständige Disziplin der Philosophie verstehen - oftmals provozierenden Gedankengänge nicht nur für eine ethische Reflexion des Verhältnisses von Politik und Moral im Allgemeinen sondern auch für eine ethische Reflexion der Aufgaben einer ethischen Politikberatung im Besonderen. Ethische Politikberatung durch sachverständige Experten wird von Politikern oftmals im Falle moralisch strittiger Fragen in Anspruch genommen und wurde im politischen System auch durch bestimmte Formen institutionalisiert (beispielweise durch den Deutschen Ethikrat [der bis 2007 ein Nationaler Ethikrat war] und durch die EnqueteKommission Ethik und Recht der modernen Medizin in der 15. Wahlperiode des Deutschen Bundestages). Der für die ethische Reflexion ethischer Politikberatung interessante Ratschlag des Systemtheoretikers Luhmann läuft darauf hinaus, dass die Aufgabe der Ethik darin liegen könnte, dem politischen System gute Gründe dafür zu liefern, wann moralische Kommunikation - als eine spezifische Weise der Kommunikation - verwendet werden sollte und wann sie unterbleiben sollte. ${ }^{3}$ Diesem Gedankengang wird im Weiteren nachgegangen werden. Zuerst werden die komplexen systemtheoretischen Überlegungen zur Unterscheidung von Moral und Ethik skizziert, die mit Blick auf die ethische Reflexion ethischer Politikberatung als kritisches Element in Stellung gebracht werden sollen. An diese Skizze schließt sich eine ebenfalls systemtheoretisch inspirierte Analyse des Gefährdungspotenzials moralischer Kommunikation an, dass sich im Rahmen einer parlamentarischen Debatte über die gesetzliche Regelung der Anwendung der Präimplantationsdiagnostik beobachten lässt. Sodann wird eine Konkretisierung der Ausführungen vorgenommen und die Unterscheidung zwischen Moral und Ethik wird im spezifischen Zusammenhang einer politikberatenden Ethikkommission - nämlich im Kontext des Nationalen Ethikrats - beobachtet. Abschließend wird mit dem ethischen Theorieansatz des irrenden Gewissens, der von Thomas von Aquin entwickelt wurde, ein komplexer ethischer Theorieansatz diskutiert, der aus der Moralfalle herausführen könnte, in der die ethische Politikberatung - zumindest aus der Perspektive der Systemtheorie - unweigerlich zu stecken scheint.

\section{Systemtheoretische Provokationen}

Niklas Luhmann hätte die Überschrift „systemtheoretische Provokationen“ sicherlich als unzulässige Etikettierung zurückgewiesen und stattdessen vermutlich von „systemtheoretischen Beobachtungen“ oder vielleicht auch von „systemtheoretischen Irritationen“ gesprochen. Die Begriffe „Moral“ und „Ethik“ betrachtet der Systemtheoretiker Luhmann mit dem ihm eigenen Argwohn, werden diese Termini seines Erachtens doch in der Regel auch in philosophischen Fachdiskursen derart inflationär bzw. unpräzise verwendet, dass der soziologische Ertrag im Sinne der Entwicklung einer Theorie der Gesellschaft dürftig ist (vgl. Luhmann, 2008 b, S. 253). Luhmann untersucht die Begriffe „Moral“ und „Ethik“ in systemtheoretischer Perspektive. Mit Blick auf die von ihm angestrebte Theorie der Gesellschaft kommt er zu dem Ergebnis, dass es zwar eine binäre Unterscheidung von "gut“ und „böse“ gibt, aber „die moderne Gesellschaft kein besonderes Funktionssystem für Moral ausdifferenziert" (Luhmann, 2008 a, S. 185) hat. Der Moralcode weist aus systemtheoretischer Perspektive eine Besonderheit auf, da „er nicht auf ein spezifisches System verweist, das allein unter diesem Code operiert und die eigenen Operationen daran erkennt. Die für die moderne Gesellschaft typische Kombination von Universalismus und Spezifikation versagt." (Luhmann, 2008 a, S. 192) Wenn die Unterscheidung von „gut“ und „böse“ nun aber nicht in allen Funktionssystemen permanent angewendet werden soll, dann muss es „in der Moral einen Einschaltungswert bzw. einen Ausschaltungswert geben, dessen Benutzung

3 Es ist zu betonen, dass Luhmann keiner feindlichen Übernahme der Ethik durch eine systemtheoretisch ausgerichtete Soziologie das Wort redet: „Die Soziologie könnte der Moral reflektierenden Ethik eine Übernahmeangebot machen und alles andere den Moralisten überlassen. Aber man kann zweifeln, ob sie damit gut beraten wäre in einer Gesellschaft, die so viel Hoffnungen auf Ethik setzt." (Luhmann, 2008 a, S. 191) Dem Systemtheoretiker Luhmann geht es auch nicht darum, die Unterscheidung Max Webers zwischen Gesinnungsethik und Verantwortungsethik aufzugreifen (vgl. Luhmann, 2008 c, S. 343 und Weber, 1988). Die Frage, ob Politiker besser ihre Gesinnung zum Maßstab ihres politischen Handelns machen oder sich stattdessen an der Frage orientieren sollten, ob das Ergebnis ihres politischen Handelns verantwortbar ist, erfasst aus systemtheoretischer Perspektive gar nicht das eigentliche Problem. Insofern kann die Lösung auch nicht darin liegen, eine Balance zwischen Gesinnungs- und Verantwortungsethik zu finden. 
darüber entscheidet, ob ein Thema moralisiert werden soll oder besser nicht. “ (Luhmann, 2008 a, S. 186) Wenn es diesen Einschaltungs- bzw. Ausschaltungswert nicht gäbe, dann könnten die Funktionssysteme nicht nach den ihnen eigenen binären amoralischen Codierungen voneinander unterschieden werden, sondern die Unterscheidung zwischen „gut“ und „böse“ würde als alleinige moralische Supercodierung angewandt oder aber in allen anderen amoralischen Codierungen eingegliedert werden. „Dann aber wäre die Gesellschaft nichts anderes als ein System der Verteilung von Achtung und Mißachtung.“ (Luhmann, 2008 a, S. 186) In einem solchen Fall bräche das Funktionssystem Politik zusammen, weil die politische Kommunikation nicht länger mittels des Mediums Macht und der Unterscheidung zwischen Regierung und Opposition reproduziert werden würde. Für das Funktionssystem Politik sind Regierung und Opposition gleichermaßen relevant. Würde nun im Funktionssystem Politik dauerhaft die Unterscheidung von „gut“ und „böse“ benutzt und sogar auf die Unterscheidung zwischen Regierung und Opposition übertragen werden, dann würde das zu einer Regression des Funktionssystems Politik führen. „Es darf gerade nicht dahin kommen, dass man die Regierung für strukturell gut, die Opposition für strukturell schlecht oder gar böse erklärt. Das wäre die Todeserklärung für Demokratie." (Luhmann, 2008 b, S. 259) Gleiches gilt übrigens auch umgekehrt: Auch die Regierung darf nicht für strukturell „böse“, die Opposition für strukturell „gut“ erklärt werden. Hinzu kommt: Würde Politikern permanent unterstellt werden, dass sie als Politiker immer schon korrupt oder aber Lügenbolde sind, dann erübrigten sich Wahlen als das wichtigste Verfahren der Legitimation des Machtbesitzes. ${ }^{4}$

Luhmann versteht „Ethik“ als „Reflexionstheorie der Moral“ und dieses Verständnis hängt mit seinem theorieinternen Ausgangspunkt der funktionalen Ausdifferenzierung der modernen Gesellschaft zusammen (vgl. Luhmann, 2008 c, S. 284). Seine Kritik am Zustand der Ethik als Reflexionstheorie der Moral setzt an seiner Beobachtung an, dass die Ethik in ihren unterschiedlichen theoretischen Facetten und disziplininternen Auseinandersetzungen bislang nur nach vernünftigen Gründen bzw. Letztbegründungsstrategien für die moralische Dignität der Unterscheidung von „gut“ und „böse“ gesucht habe. „Die Ethik sucht (sofern sie ihrer Tradition treu bleiben will) gute Gründe dafür, dass es gut sei, die Unterscheidung gut/böse als Form moralischer Kommunikation zu verwenden.“ (Luhmann, 2008 a, S. 190) Die Ethik urteilt also mit einem moralisierend-appellativen Charakter über die Moral. Sie nimmt somit „zugleich eine moralische Selbstbewertung “ (Luhmann, 2008 a, S. 177) vor, Ethik kann sich so „selbst als moralisches Unternehmen begreifen, sich in ihre eigene Beschreibung der Moral einbeziehen und, vereinfacht gesagt, sich selbst für gut halten “ (Luhmann, 2008 b, S. 258). Mit der Hilfe der moralischen Selbstbewertung entlässt sich die Ethik selbst aus der Pflicht, eine weitere binäre Codierung einzuführen. Moral bzw. moralgeladene Kommunikation ist aus der Perspektive der Ethik "gut“ und nicht „böse“. Das Problem einer solchen Reflexionstheorie der Moral liegt für Luhmann darin, dass sie den universellen Geltungsanspruch der moralgeladenen Kommunikation unterstützt, ja geradezu befeuert. Und das obwohl sich soziologisch durchaus das Phänomen entdecken lasse, dass „die Moral vielerlei Gebrauch von Moral moralisch missbilligt“ (Luhmann, 2008 a, S. 186). Das geschieht, wenn die Moralisierung von bestimmten Handlungen als unmoralisch bzw. zumindest unangemessen identifiziert werden sollte. Während die moralgeladene Kommunikation also im Rahmen ihrer eigenen Möglichkeiten (nämlich mit der Hilfe moralgeladener Kommunikation) den universalistischen Anspruch infrage stellt, gibt die Ethik als Reflexionstheorie der Moral diesen universalistischen Anspruch nicht auf.

Die eigentliche (systemtheoretisch motivierte) Frage an die Ethik stellt sich somit angesichts der universalistischen Ausrichtung der Moral, die ohne eine weitere Spezifikation daherkommt (vgl. Luhmann, 2008 a, S. 192) und genau diese Spezifikation zumindest im Sinne des oben angesprochenen Einschaltungs- bzw. Ausschaltungswerts doch benötigt. Es geht Luhmann um eine Ethik als Reflexionstheorie der Moral, die sich bezüglich der Beurteilung der Moral nicht einfach der Codierung „gut“ versus „böse“ bedient, sondern „die die Probleme und die Risiken des Moralisierens kennt und berücksichtigt" (Luhmann, 2008 a, S. 194) und mit einer systemtheoretisch fundierten Gesellschaftstheorie kooperiert.

Auch wer die Beobachtungen des Systemtheoretikers nachzuvollziehen bereit ist und die derart analysierte Eigenart der moralischen Kommunikation erkennt, der steht - beispielsweise als Ethiker - vor einem Problem. Denn die systemtheoretischen Beobachtungen offenbaren ja gerade, dass sich die moralische Kommunikation in den unterschiedlichen Funktionssystemen nicht einfach abstellen lässt - auch nicht mit der Hilfe wohlgesinnter systemtheoretisch informierter Ethiker. Die systemtheoretische Analyse eröffnet einen spezifischen Problemzugang, sie stellt aber noch lange keinen binären Code für die Ethik als Reflexionstheorie der Moral zur Verfügung, mit dem die Ethik als Reflexionstheorie der Moral dann operieren könnte. Im Gegensatz zu konventionellen ethischen Theorieansätzen ignoriert der systemtheoretisch informierte Ethiker das Problem der Moral zwar nicht; und weil er einen Sinn für die Eigenart des Problem hat, solidarisiert er sich auch nicht mit der moralgeladenen Kommunikation. Aber aus den systemtheoretischen Überlegungen lässt sich keine neue Ethik ableiten. „Man sieht zwar, dass eine Ethik, wenn sie denn eine Reflexionstheorie moralischer Kommunikation sein will, heute andere Probleme zu lösen hat als zu Lebzeiten des »Subjekts«. Man sieht aber nicht, welche Formen der Entfaltung der Unterscheidungsparadoxie der Moral als Ethik angeboten werden könnten.“ (Luhmann, 2008 a, S. 194) Allerdings geht

4 Diese Warnung vor der Moralisierung der amoralischen Codierungen der Funktionssysteme der modernen ausdifferenzierten Gesellschaft hält Luhmann aber nicht davon ab, nach der funktionalen Bedeutung der Moral im Funktionssystem Politik zu fragen, schließlich ignoriert die moralgeladene Kommunikation „die sonst im System gebräuchlichen Unterscheidungen“ (Luhmann, 2008 a, S. 186). 
es dem Soziologen und Systemtheoretiker Luhmann auch nicht um eine theoretische Entwicklung einer wissenschaftlichen Ethik bzw. um die Begründung der Moral, sondern um die Beobachtung der Ethik (vgl. Luhmann, 2008 c, S. 271). „Ob eine Ethik überhaupt möglich ist, die die Verhältnisse in der modernen Gesellschaft berücksichtigt und sich selber für gut erklärt, kann man als Soziologe nicht abschließend beurteilen.“ (Luhmann, 2008 b, S. 266-267)

\section{Das Gefährdungspotenzial moralischer Kommunikation}

Wenn der Ethiker als Reflexionstheoretiker die Moral beobachtet, dann hat er festzustellen, dass moralgeladene Kommunikation nicht permanent eingesetzt wird: „Nur wenn es brenzlig wird, hat man Anlaß, die Bedingungen anzudeuten oder gar explizit zu nennen, unter denen man andere bzw. sich selbst achtet oder nicht achtet.“ (Luhmann, 2008 b, S. 257) Um diesen Gedankengang zu verdeutlichen, soll ein Blick in die parlamentarische Debatte über die gesetzliche Regelung der Anwendung der Präimplantationsdiagnostik geworfen werden. Die Abstimmung am 7. Juli 2011 im Deutschen Bundestag wurde im Vorfeld zu einer heiklen Moralfrage bzw. moralischen Grundsatzfrage deklariert und verschiedene Ethik-Experten wurden als Politikberater eingeschaltet. ${ }^{5}$ Moralfragen werden immer auch als Gewissensfragen verhandelt, deshalb wird der (informelle) Fraktionszwang aufgehoben und - aus moralischen Gründen - auf die grundgesetzlich verbürgte Freiheit des Abgeordnetenmandats verwiesen. Der gewählte Abgeordnete, so wird im Zusammenhang von Gewissensentscheidungen betont, übt sein Mandat im Parlament frei aus und ist niemandem gegenüber verantwortlich. Er kann bzw. soll dann unabhängig von einer - in solchen Fällen meist auch gar nicht in der gewohnten Form vorhandenen - Parteilinie, nur seinem Gewissen entsprechend entscheiden. ${ }^{6}$

Der Deutsche Bundestag hatte am 7. Juli 2011 eine vierstündige Debatte geführt und im Anschluss über drei vorliegende Gesetzentwürfe fraktionsübergreifender Parlamentariergruppen abgestimmt. Zwar werden solche Debatten um Moralfragen einerseits als „eindringlich“, „ernsthaft“, „leidenschaftlich “ und „emotional“ bezeichnet, aber andererseits dürfen sie offensichtlich auch nicht zu moralisch geführt werden. Es soll auf eine moralgeladene Kommunikation verzichtet werden, die die Position des politischen Gegners als unmoralisch ausweist und damit Missachtung der Person signalisiert. ${ }^{7}$ Es wird offensichtlich akzeptiert, wenn der Abgeordnete Peter Hintze es für „,rechtlich nicht haltbar und moralisch verwerflich “ hält, die Präimplantationsdiagnostik abzulehnen und somit die „Vermeidung von Abtreibung zu verbieten“. Aber ein Redakteur der Frankfurter Allgemeinen Zeitung thematisierte in einem Artikel am 8. Juli 2011 die Kommunikation zweier Abgeordneten, die die Präimplantationsdiagnostik befürworteten. Offensichtlich hatten sich die Abgeordneten Karl Lauterbach und Kerstin Müller allzu moralisch geäußert: Lauterbach „bezweifelte, dass es sich beim Embryo in der Petrischale bereits um einen Menschen handelt. Im Übrigen warf er den Verbotsanhängern vor, sie unterstellten den PIDBefürwortern eine mangelnde Liebe zum Kind. Das war eine der wenigen Situationen, wo es einmal lauter wurde und Zwischenrufe aus dem Halbrund des Plenarsaals Richtung Sprecherpult geschleudert wurden. Wenig später sollte die Grüne Kerstin Müller den Verbotsanhängern unterstellen, sie führten ein Nachhutgefecht zur Regelung über den Schwangerschaftsabbruch. So viel Schärfe war selten.“ (Mihm, 2011, S. 2)

Anhand dieses kleinen Debattenausschnitts lässt sich zeigen, dass die moralgeladene Kommunikation ein Gefährdungspotenzial mit sich bringt (vgl. Luhmann, 2008 c, 280). „Denn praktisch gehen Moralisten davon aus, dass sie es mit Gegner zu tun haben, die nicht überzeugt werden können. Die Moral nimmt damit kämpferische Züge an. Sie entflammt sich geradezu am Konflikt und begründet ihren Standpunkt mit Hilfe der Ablehnung einer anderen Einstellung, die sie in der Gesellschaft vorfindet.“ (Luhmann, 2008 a, S. 193) Sie bringt nicht nur das Risiko mit sich, dass sie gegen den Autor bzw. die Autorin der Kommunikation selbst gewendet werden kann (die Verbotsbefürworter der Präimplantationsdiagnostik könnten den Verbotsgegner dann vorwerfen, dass diese ihnen eine mangelnde Rücksichtnahme des Leides der Eltern unterstellten oder aber [sic!] ein Nachhutgefecht zur Regelung über den Paragraf 218 des Strafgesetzbuches führten); moralgeladene Kommunikation „führt im Ausdruck von Achtung und Missachtung zu einem Überengagement der Beteiligten“ (Luhmann, 2008 b, S. 260). Dann aber stehen die überengagierten Politiker vor dem Problem, dass sie mit den von ihnen moralisch Verurteilten weiter umgehen müssen. Auch in anderen politischen Fragen müssen weiterhin Mehrheiten organisiert werden! Welche Lehren lassen sich aus diesem Beispiel für eine ethische Politikberatung ziehen, die das politische System bei der Entscheidungsfindung in moralisch strittigen Fragen unterstützen soll?

5 Bundestagsdebatten zu moralischen Problemen werden mittlerweile (oftmals bereits bevor sie stattgefunden haben) von Politikern und Journalisten als „Sternstunden des bundesdeutschen Parlamentarismus" bezeichnet.

6 Das freie Mandat der Mitglieder des Deutschen Bundestages ist in Art. 38 Abs. 1 Satz 2 des Grundgesetzes verankert. Hier heißt es, dass die Abgeordneten „Vertreter des ganzen Volkes [sind], an Aufträge und Weisungen nicht gebunden und nur ihrem Gewissen unterworfen." Aus demokratietheoretischer Perspektive ist festzuhalten, dass diese Gewissensfreiheit nicht nur im Kontext der von den Parteispitzen identifizierten moralischen Fragen seine Geltung beansprucht, sondern in allen politischen Fragen. Allerdings führt eine konsequente Moralisierung der parlamentarischen Entscheidungsprozesse mit Rekurs auf den moralisch konnotierten Begriff des Gewissens zu mannigfaltigen Problemen. Denn entweder wird die moralgeladene Kommunikation auf Dauer gestellt oder aber es muss den von Luhmann erwähnten Einschaltungswert bzw. Ausschaltungswert geben: Wann handelt es sich um eine Gewissensentscheidung und wann nicht?

7 Für Luhmann zeigt sich ein Entscheidungsparadox: „Gerade weil Moralfragen unentscheidbar sind, kann und muss man sie entscheiden. Nur kann man dann nicht vermeiden, dass die Entscheidungen als Entscheidungen und das heißt: als auch anders möglich, sichtbar werden und dass ihnen eine letzte, hieb- und stichfeste Begründung fehlt." (Luhmann, 2008 a, S. 178) Für das politische System zeigt sich, dass auch die Abstimmung über moralische Fragen trotz Aufhebung des Fraktionszwangs in der binären Codierung Regierung/Opposition bzw. Überlegene/Unterlegene verbleibt. Es gibt nach einem Wahlgang immer eine Mehrheit und eine Minderheit. 


\section{Konkretisierung: Zum Verhältnis von Moral und Ethik in Ethikkommissionen}

Welchen Ertrag haben die bisherigen Ausführungen für eine ethische Reflexion einer ethischen Politikberatung (vgl. zu den Besonderheiten bzw. Selbstbeschreibungen einer ethischen Politikberatung Lob-Hüdepohl, 2006; Weilert, 2011; Weilert \& Hildmann, 2012), die sich im Sinne Luhmanns als eine Reflexionstheorie der moralgeladenen Kommunikation im Funktionssystem Politik Gehör verschaffen will? Es lässt sich allgemeinhin feststellen, dass eine ethische Politikberatung beispielsweise in der institutionell bekanntesten Form einer Ethikkommission - im politischen System häufig dann herangezogen wird, wenn bestimmte parlamentarische Diskussionsund Abstimmungsprozesse anstehen und diese als moralisch bedeutsam ausgewiesen werden. Die Legitimität von Ethikkommissionen ist aus unterschiedlichen moralischen und rechtlichen Perspektiven problematisiert worden (vgl. Graumann, 2007; Riedel, 2005; Schicktanz \& Schweda, 2007; Vöneky, 2009; Zotti, 2009). Moralisch unstrittig ist, dass Ethiker - auch aus der Perspektive einer Reflexionstheorie der Moral - nicht dazu berufen sind, auf moralische Fragen letztgültige Antworten zu geben. Sie können auch aus demokratietheoretischer Perspektive nicht an Stelle der Abgeordneten sprechen. ${ }^{8}$

Wie aber lässt sich die Konjunktur der Ethikkommissionen aus systemtheoretischer Sicht erklären, welche funktionale Bedeutung haben Ethikkommissionen für das Funktionssystem Politik? Luhmanns systemtheoretischer Antwortversuch: Sie sind kommunikative Arrangements und können „als eine Parallelaktion zu den Kontingenten und Unabwägbarkeiten, den Karrierespielen und den Zustimmungskalkülen der Parteiendemokratie begriffen werden, als ein zweiter Weg der Problembearbeitung, der Reduktion von Ungewissheit, der Konsenssuche. Der achtungsgebietende Begriff der Ethik hat dann die Funktion, Distanz zu legitimieren und zugleich den Anschein zu pflegen, als ob es nicht um Interessen ginge. So verstanden gehört Ethik mit zur höheren Amoralität einer demokratischen politischen Kultur." (Luhmann, 2008 a, S. 195) In derart verstandenen Ethikkommissionen gehöre es zudem zu der Bedingung der Interaktion, „dass die Beteiligten davon absehen, wechselseitig Achtung oder Missachtung aufs Spiel zu setzen“ (Luhmann, 2008 a, S. 195).

Diese Annahmen Luhmanns decken sich mit der reflektierenden Berichterstattung des Soziologen Wolfgang van den Daele. ${ }^{9}$ Dieser geht davon aus, dass der Nationale Ethikrat mittlerweile abgelöst durch den Deutschen Ethikrat - als ein politisch beauftragtes, institutionalisiertes Gremium der argumentativen Erwägung und kommunikativen Aushandlung dazu beitragen sollte, virulente moralische Konflikte, die im Zusammenhang mit den Bio- bzw. Lebenswissenschaften auftreten, im Vorfeld parlamentarischer Beratungen und Abstimmungen zu entschärfen. ${ }^{10}$ Van den Daele hält fest, dass die Kommunikation der Mitglieder des Nationalen Ethikrats nie entgleist sei, obwohl eine moralgeladene Kommunikation stets präsent gewesen sei und in der Mehrzahl der verhandelten moralischen Streifragen kein moralischer Konsens hergestellt werden konnte, sondern moralischer Dissens die Regel gewesen sei. Trotzdem habe kein Mitglied des Nationalen Ethikrates „die Trompete des Krieges geblasen“ (van den Daele, 2008, S. 362), der Rubikon wurde offensichtlich nie überstritten. Das ist bemerkenswert, weil die Mitglieder „selbst Parteien im bioethischen Streit und damit bestrebt waren, sich in diesem Streit moralisch klar zu positionieren " (van den Daele, 2008, S. 371). Diese moralische Parteilichkeit schien vom politischen System gewünscht zu sein, weil damit der moralische Konflikt in einem öffentlichen Streit abgebildet wurde. Allerdings bestand das Risiko, dass sich die Mitglieder des Nationalen Ethikrates wechselseitig als Personen hätten missachten können und ein Mitglied den Rat mit dem Hinweis auf die dort vertretenen inakzeptablen moralischen Positionen verlässt.

Die Befriedung der moralischen Streitfragen bzw. die Einhegung moralgeladener Kommunikation wird mit dem gewählten deliberativen bzw. diskursiven Verfahren begründet, das für Ethikkommissionen allgemeinhin favorisiert wird. Dieses Verfahren „erzeugt eine Sachlichkeit der Kommunikation, die persönliche Polemik ausschließt und der Eskalation der Konflikte entgegenwirkt“ (van den Daele, 2008, S. 362). Die Moral des deliberativen Verfahrens fordert nämlich von allen am Diskurs Beteiligten, dass sie moralische Dissidenten nicht missachten, sondern ihnen trotz der moralisch differen-

8 Insofern ist ethische Politikberatung nicht mit einer advokatorischen Ethik zu verwechseln. Im Kontext der moralischen Debatten um die technischen Eingriffe an Embryonen kann sich aber die Frage nach der ethischen Legitimität einer solchen advokatorischen Ethik zu stellen. Vgl. dazu - frei von systemtheoretischen Irritationen - Bohmeyer 2012.

9 Die Reflexionen des Soziologen Wolfgang van den Daele beziehen sich ausschließlich auf den „Nationalen Ethikrat“, dem er von 2001 bis 2007 als Mitglied angehört hat. Im Anschluss an Luhmann geht van den Daele davon aus, dass moralischen Streitfragen „eine Tendenz zur Unnachgiebigkeit und Unversöhnlichkeit eigen“ (van den Daele, 2008, S. 358) ist. „Moral ist per definitionem intolerant; sie macht kriegerisch." (van den Daele, 2008, S. 360).

10 Die Institutionalisierung des Deutschen Ethikrats ist im Gesetz zur Einrichtung des Deutschen Ethikrats (Ethikratgesetz - EthRG) geregelt, das am 16. Juli 2007 ausgefertigt wurde (BGBI. I S. 1385) und am 1. August 2007 in Kraft getreten ist. Nach diesem Gesetz (vgl. §2, Abs. 1) gehört es nicht nur zu den Aufgaben des Deutschen Ethikrats eine ethische Reflexion vorzunehmen, sondern auch die „gesellschaftlichen, naturwissenschaftlichen, medizinischen und rechtlichen Fragen sowie die voraussichtlichen Folgen für Individuum und Gesellschaft [zu verfolgen], die sich im Zusammenhang mit der Forschung und den Entwicklungen insbesondere auf dem Gebiet der Lebenswissenschaften und ihrer Anwendung auf den Menschen ergeben“. Auch setzt sich der „unabhängige Sachverständigenrat“ nicht nur aus Ethikern zusammen, sondern er „besteht aus 26 Mitgliedern, die naturwissenschaftliche, medizinische, theologische, philosophische, ethische [sic!], soziale, ökonomische und rechtliche Belange in besonderer Weise repräsentieren“ und die „Empfehlungen für politisches und gesetzgeberisches Handeln“ ( $\$ 2$, Abs. 1) geben sollen. Die ethische Expertise ist demnach nur eine Expertise unter anderen. In der Kabinettsvorlage zur Einrichtung eines Nationalen Ethikrates vom 25. April 2001 wurde die ethische Perspektive nicht eigens hervorgehoben (vgl. §3, Abs. 1). Auch die Aufgabenbeschreibung war aus der Perspektive ethischer Politikberatung eindeutiger: „Der Nationale Ethikrat nimmt Stellung zu ethischen Fragen neuer Entwicklungen auf dem Gebiet der Lebenswissenschaften sowie zu deren Folgen für Individuum und Gesellschaft." (Vgl. §2, Abs. 2). 
ten Position eine moralische Integrität zusprechen. ${ }^{11}$ Mit der moralischen Vorentscheidung der am Verfahren Beteiligten, sich auf das deliberative bzw. diskursive Verfahren einzulassen, konnten die Mitglieder des Nationalen Ethikrates die jeweiligen moralischen „Gegner“ nicht mehr dämonisieren und den verhandelten moralischen Konflikt „nicht mehr plausibel als einen Zusammenstoß der Guten mit den Bösen stilisieren " (van den Daele, 2008, S. 364). Zudem verfolgten die beteiligten Personen im Rahmen des diskursiven Verfahrens die Strategie, „immer dann, wenn es argumentativ »gefährlich « wurde, die gemeinsame Beratung, also die interaktive Prüfung von Argumenten und Gründen, zu beenden und stattdessen mit der Ausarbeitung getrennter Stellungnahmen zu beginnen, um die eigene Position »im Zusammenhang « darzulegen. Gefährlich wurde es, wenn eingespielte moralische Begründungen ins Wanken gerieten oder unauflöslicher Dissens aufbrach, der eigentlich dazu genötigt hätte, in Diskussionen über die Geltungsbedingungen moralischer Ansprüche und über den möglichen Wandel solcher Ansprüche einzutreten. “ (van den Daele, 2008, S. 371)

Im Nationalen Ethikrat wurden also die im öffentlichen Diskurs vorfindlichen moralischen Positionen dargestellt und argumentativ untermauert. Ethische Diskussionen über die verwendeten moralischen Begründungsfiguren wurden hingegen nicht geführt, die oben angeführte Diskurstheorie der Moral wurde nicht im Sinne eines ethischen Begründungsdiskurses hinterfragt, sondern moralisch anerkannt. Außerdem wurde moralisch anerkannt, dass der Gesetzgeber in einem demokratisch verfassten Gemeinwesen in moralischen Streitfragen eine für alle Bürger - wenn schon nicht moralisch, dann aber zumindest juristisch - bindende Gesetzesgrundlage schaffen würde, selbst wenn diese sich nicht mit dem Votum des Nationalen Ethikrates decken würde. Es wurde von den Mitglieder des Nationalen Ethikrates moralisch anerkannt und nicht im Sinne einer ethischen Reflexion hinterfragt, dass der moralische Dissens bezüglich unterschiedlicher moralischer Fragen der Bio- bzw. Lebenswissenschaften innerhalb des diskursiven Verfahrens nicht aufgelöst werden konnte. Das politische System und das heißt das demokratische Verfahren der Mehrheitsbeschaffung wurde dann zur politischen Auflösung des moralischen Konflikts angefragt.

Die Lösung oder Befriedung des moralischen Streits wurde also an das politische System rücküberwiesen, das doch selbst um moralische Expertise gebeten hatte. Die moralische Befangenheit der berufenen Ethiker bezüglich der Beurteilung der virulenten moralischen Fragen und ihre moralische Festlegung auf das diskursive Verfahren führten dazu, dass die Ethik nicht im Sinne einer Reflexionstheorie der Moral, sondern ausschließlich im Sinne einer argumentativen Explikation der eigenen moralischen Position betrieben wurde. Das ehemalige Mitglied van den Daele konstatiert: Die moralische Parteilichkeit der Mitglieder „hat ihre Bereitschaft begrenzt, im Diskurs von moralischer Kommunikation zur Kommunikation über Moral zu wechseln, also moralische Geltung in der Argumentation nicht als Ressource, sondern als Problem zu behandeln.
Am Ende einer solchen Problematisierung hätte als gemeinsames Eingeständnis moralische Ratlosigkeit, Nichtentscheidbarkeit oder eben die abstrakt beschworene »epistemische Demut « stehen können. “ (van den Daele, 2008, S. 373) Stattdessen wurde der moralische Dissens durch die Abgabe unterschiedlicher Stellungnahmen und Voten dokumentiert und der moralische Konflikt dann wieder dem Verfahren der demokratischen Mehrheitsentscheidung überantwortet. ${ }^{12}$ „Demokratie schlägt Moral. Dieses Signal bricht dem moralischen Dissens gewissermaßen die fundamentalistische Spitze ab. “ (van den Daele, 2008, S. 378)

Offensichtlich nutzte das politische System den Nationalen Ethikrat und nutzt nun den Deutschen Ethikrat, um moralisch brisante parlamentarische Beratungsgegenstände im Vorfeld der parlamentarischen Diskussion zu diskutieren. Wenn dieses Gremium dann die Beratung zurückgibt, ohne einen Konsens hergestellt zu haben, dann ist ein der moralische Konflikt schon ein wenig entschärft und das Gefährdungspotenzial der moralischen Kommunikation für die politischen Interaktionsprozesse wurde eingedämmt.

\section{Ausweg aus der Moralfalle}

Was aber bleibt nun einer ethischen Politikberatung, die nicht wie im oben skizzierten Beispiel des Nationalen Ethikrats in der eigenen moralischen Befangenheit verharrt, sondern die als Reflexionstheorie der Moral agieren will? Das soziologisch analysierte Gefährdungspotenzial der moralgeladenen Kommunikation lässt Luhmann zu dem Schluss kommen, dass die Ethik als Reflexionstheorie der Moral versuchen sollte, „den Anwendungsbereich von Moral zu limitieren“ (Luhmann, 2008 b, S. 266). Solange sie aber keine verbindlichen Kriterien für die Begrenzung der moralischen Kommunikation benennen könne, solange „ist es die vielleicht vordringlichste Aufgabe der Ethik, vor Moral zu warnen“ (Luhmann, 2008 b, S. 266; vgl. dazu auch Luhmann, 2008 c, S. 346).

Sofern man eine ethische Reflexion politischer Vorgänge überhaupt für wichtig hält, muss aber eben doch eine Form für eine ethische Politikberatung gefunden werden, die die zuvor skizzierten systemtheoretische Beobachtungen der Moral aufgreift und mit der moralischen Kommunikation im politischen System umzugehen weiß. Die folgenden Ausführungen lösen vielleicht den von Luhmann formulierten theoretischen Anspruch ein, dass der folgende ethische Theorieentwurf „sich nicht einfach mit der guten Seite der Moral solidarisiert und die schlechte vergisst, sondern die Moral als eine Unterscheidung thematisiert, nämlich als die Unterscheidung von gut und schlecht oder gut und böse." (Luhmann, 2008 b, S. 267).

Ausgerechnet die mittelalterliche Theologie könnte der Ethik bzw. ethischen Politikberatung im nachmetaphysischen

11 Vgl. zu einer Diskurstheorie der Moral insbesondere die von Jürgen Habermas und Karl-Otto Apel entwickelte Theoriearchitektur (vgl. Habermas, 1991).

12 Vgl. zur ethischen Reflexion des Begriffspaars moralischer Konsens/ moralischer Dissens Bayertz, 1996 a und Bayertz, 1996 b. 
und zugleich postsäkularen Zeitalter einen (auch systemtheoretisch interessanten) Ausweg aus der Moralfalle bieten, weil mit der „änigmatische Idee“ (Hoye, 1996, S. 420) des irrenden Gewissens von Thomas von Aquin ein komplexer ethischer Theorieansatz zur Verfügung gestellt wird, der zur Entparadoxierung moralischer Kommunikation (vgl. Luhmann, 2008 c, S. 294) herangezogen werden kann. ${ }^{13}$

Der scholastische Theologe und Philosoph Thomas von Aquin widmet sich in seiner Summa theologiae verschiedenen Fragestellungen, die das moralische Handeln von Personen betreffen. ${ }^{14}$ Im Kontext seiner Auseinandersetzung mit dem Begriff des Gewissens, stellt Thomas die Frage, ob ein von einer irrenden Vernunft abweichender Wille einer Person moralisch schlecht ist bzw. ob ein im Irrtum befindliches Gewissen den Träger dieses Gewissen moralisch bindet? Er kommt zu dem Ergebnis, dass eine Person im Falle eines irrenden Gewissens - selbst wenn es sich um einen selbstverschuldeten Irrtum handelt - aus moralischen Gründen verpflichtet ist, dem Gewissen zu folgenden und somit das Falsche zu tun. ${ }^{15}$ „Im Falle eines irrenden Gewissens ist es einem nicht nur erlaubt, das Falsche zu tun; man ist sogar dazu verpflichtet. Hier gestattet Thomas keine Kompromisse, auch nicht, wenn der Irrtum selbst verschuldet ist. Insofern geht er über die von der Katholischen Kirche übernommene Ansicht hinaus, wonach nur ein schuldlos irrendes Gewissen binde." (Hoye, 1996, S. 423) Die Bezugnahme auf das irrende Gewissen soll den Träger eines solchen Gewissens nicht von seiner moralisch falschen Handlung entschuldigen, stattdessen wird die Verpflichtung zur moralischen Selbstbindung betont. Mit Blick auf den modernen Autonomiebegriff nimmt Thomas mit seinem - in gewisser Weise schon subjektorientierten - Gewissensbegriff das Wesen der Moralität bzw. die moralische Integrität der Person in den Blick. Diese moralische Integrität einer Person gilt es unbedingt zu schützen, auch wenn die Entscheidung bzw. Handlung einer solchen Person aus der Perspektive eines nicht-irrenden Gewissens als unmoralisch ausgewiesen werden muss. Der Träger eines irrenden Gewissens muss diesem gehorchen, da sonst gegen die Wurzel seiner eigenen Sittlichkeit verstößt.

Thomas unterscheidet also die moralische Qualität des Willens einer Person von der moralischen Qualität der Handlung, die diese Person vollzieht. Im Sinne einer solchen Unterscheidung kann eine Gewissensentscheidung nicht als solche böse bzw. schlecht sein, allerdings kann die resultierende Handlung durchaus (von den Personen mit nicht-irrendem Gewissen) als böse bzw. schlecht bewertet werden. Außerdem verlangt das Gewissen nach einer selbstkritischen (,gewissenhaften“) Prüfung. Wer sich auf sein Gewissen beruft, muss begründet darlegen können, dass das als moralisch „gut“ Erkannte auch „gut“ und nicht „böse“ ist. Insofern besteht eine Bildungspflicht des Gewissens, aber nach dieser Pflicht zur Ausbildung des Gewissens besteht für die Person - als moralische Person - eine Bindungspflicht. Sofern eine Person einem Irrtum wissentlich folgt, handelt es sich ebenfalls um eine unmoralische Handlung (vgl. Sth I-II, 19,6). In einem solchen Fall kann man gar nicht von einem irrenden Gewissen sprechen. Ein irrendes Gewissen ist ausschließlich ein solches, das um seinen Irrtum nicht weiß.

Obwohl die Verantwortung für die Ausbildung des Gewissens und für seine Anwendung nicht an Ethiker delegiert werden kann, sollte sie erst recht nicht den Moralisten bzw. Inquisitoren überlassen werden. Der ethische Theorieentwurf des irrenden Gewissens gibt einer ethischen Politikberatung durchaus die Möglichkeit an die Hand, mit Blick auf die moralischen Streitfragen an der Bildungspflicht des Gewissens der Politiker teilzunehmen. Aber eine solche Bezugnahme verpflichtet eine ethische Politikberatung, auf die moralische Integrität der beteiligten Personen zu verweisen und mit Blick auf die Möglichkeit des irrenden Gewissens eine epistemische Demut in Stellung zu bringen. „Menschen, die mit einem individuell beschränkten Gewissen ausgestattet sind, erfreuen sich nicht der Augen platonischer Adler, die laut einer mittelalterlichen Legende das Vermögen besitzen, direkt in die Sonne zu schauen; für Thomas sind wir eher wie aristotelische Eulen, die in einer schattenhaften Realität unterwegs sind. Der Einsicht in die ambivalente Individualität dieser condition humaine und ihrer politischen Anerkennung in der Neuzeit verdanken wir das demokratische Prinzip der Gewissensfreiheit." (Hoye, 1996, S. 435) Einer mit dem ethischen Theorem des irrenden Gewissens operierenden ethischen Politikberatung geht es dann in erster Linie um die Wahrung der moralischen Integrität der Beteiligten Personen und im Sinne Luhmanns um eine Begrenzung einer personenbezogenen Moralisierung. Die moralgeladene Kommunikation kann durch die moralische Berufung auf das Bewertungsdefizit des irrenden Gewissens entschärft werden. Wer sich auf das irrende Gewissen beruft, schaltet die moralische Kommunikation (zumindest teilweise) ab. Moralischer Dissens kann dann zwar nicht mit Blick auf die verhandelte moralische Streitfrage, aber mit

13 Vgl. zur Einführung in den Begriff des „irrenden Gewissens“ (Hoye, 1996; sowie Heinzmann, 1990 und Heinzmann, 1991). Mir ist bekannt, dass der Begriff des irrenden Gewissens in der Ethik umstritten ist, insbesondere im Kontext der unterschiedlichen Rezeptionen der Kantischen und Fichte'schen These, die das irrende Gewissen als eine Unmöglichkeit darstellen. Insbesondere die Philosophie der Aufklärung konnte die Idee eines irrenden Gewissens nicht akzeptieren. (Vgl. dazu auch Spaemann, 2001).

14 Vgl. Sth. I-II q. 18-21. Zitiert nach Thomas von Aquin, 2001.

15 Die Katholische Kirche hat den Begriff des irrenden Gewissens des Kirchenlehrers aufgegriffen und im Katechismus der Katholischen Kirche (vgl. Katechismus der Katholischen Kirche, 2007, dritter Teil, Art. 179off) einige moraltheologische Erwägungen angestellt. Im Gegensatz zu Thomas von Aquin wird hier sehr stark auf die Verantwortlichkeit der Person zur Ausbildung des Gewissens verwiesen und auf die selbstverschuldete Unkenntnis des Gewissens verwiesen. Als Grund von Fehlurteilen des Gewissens wird zudem auf den „Anspruch auf eine falsch verstandene Gewissensautonomie“ verwiesen. Damit wird der in gewisser Weise bereits subjektorientierte Ansatz des Thomas eingeschränkt und sein mit dem Gewissen eingeführtes Individualitätsprinzip wieder eingeschränkt. Es gilt aber: „Wenn hingegen die Unkenntnis unüberwindlich oder der Betreffende für das Fehlurteil nicht verantwortlich ist, kann ihm seine böse Tat nicht zur Last gelegt werden. Trotzdem bleibt sie etwas Böses, ein Mangel, eine Unordnung. Aus diesem Grund müssen wir uns bemühen, Irrtümer des Gewissens zu beheben." (1793). 
Blick auf die zu wahrende moralische Integrität der beteiligten Personen moralisch akzeptiert werden ${ }^{16}$

\section{Literatur}

Bayertz, K. (1996a). Einleitung. Moralischer Konsens als soziales und philosophisches Problem. In: Bayertz, K. (Hrsg.). Moralischer Konsens. Technische Eingriffe in die menschliche Fortpflanzung als Modellfall. Frankfurt am Main: Suhrkamp Verlag, S. 11-29.

Bayertz, K. (1996 b). Moralischer Konsens. Überlegungen zu einem ethischen Grundbegriff. In: Bayertz, K. (Hrsg.). Moralischer Konsens. Technische Eingriffe in die menschliche Fortpflanzung als Modellfall. Frankfurt am Main: Suhrkamp Verlag, S. 60-79.

Bohmeyer, A. (2012). Wer spricht für wen? Eine moralphilosophische Reflexion anwaltschaftlicher Ethik- und Politikberatung in bioethischen Kontexten. In: Weilert, A. K. \& Hildmann, P. W. (Hrsg.). Ethische Politikberatung. Baden-Baden: Nomos Verlagsgesellschaft, S. 167-181.

Geyer, C. (13. September 2011). Wie die PID so der Euro? Warum das Gewissen nicht die Probleme der Politik löst. Frankfurter Allgemeine Zeitung, Nr. 213, S. 37.

Graumann, S. (2007). Politische Ethikberatung zwischen allen Stühlen Beitrag zur ethischen Urteilsbildung oder pure Akzeptanzbeschaffung? In: Porz R. \& Rehmann-Sutter, C. \& Leach Scully, J. \& Zimmermann-Acklin, M. (Hrsg.). Gekauftes Gewissen. Die Rolle der Bioethik in Institutionen. Paderborn: Mentis, S. 281-290.

Habermas, J. (1991). Erläuterungen zur Diskursetbik. Frankfurt am Main: Suhrkamp Verlag.

Hebel, S. (02. Januar 2012). Moralisch gescheitert. Berliner Zeitung, Nr. 1, S. 4.

Heinzmann, R. (1990). Der Mensch als Person. Zum Verständnis des Gewissens bei Thomas von Aquin. In: Gründel J. (Hrsg.). Das Gewissen. Subjekte Willkür oder oberste Norm. Düsseldorf: Patmos Verlag, S. 3452 .

Heinzmann, R. (1991). Thomas von Aquin und die Autonomie der Vernunft. In: Kutschki, N. (Hrsg.). Der Streit um den rechten Glauben. Zürich: Benziger Verlag, S. 169-183.

Hoye, W. J. (1996). Die Wahrheit des Irrtums. Das Gewissen als Individualitätsprinzip in der Ethik des Thomas von Aquin. In: Aertsen, J. A. \& Speer, A. (Hrsg.). Individuum und Individualität im Mittelalter (Miscellanea Mediaevalia, XXIV). Berlin: de Gruyter, S. 419-435.

Katechismus der Katholischen Kirche (2007). Neuübersetzung aufgrund der Editio typica Latina. Oldenbourg: St. Benno-Verlag/Paulusverlag.

Lob-Hüdepohl, A. (2006). Nicht vom hohen Ross herab. Politikberatung durch ethische Ideenagenturen. In: Herder Korrespondenz, 60. Jg., Heft 2, S. 79-83.

Luhmann, N. (2008 a). Politik, Demokratie, Moral. In: Die Moral der Gesellschaft. Frankfurt am Main: Suhrkamp Verlag, S. 175-195.

Luhmann, N. (2008 b). Paradigm Lost: Über die ethische Reflexion der Moral. In: Die Moral der Gesellschaft. Frankfurt am Main: Suhrkamp Verlag, S. 253-269.

Luhmann, N. (2008c). Ethik als Reflexionstheorie der Moral. In: Die Moral der Gesellschaft. Frankfurt am Main: Suhrkamp Verlag, S. 270347.

Mihm, A. (08. Juli 2011). Momente der Stille, Augenblicke der Empörung. Frankfurter Allgemeine Zeitung, Nr. 156, S. 2.

Müller-Vogg H. \& Wulff C. (2007). Besser die Wahrheit. Hamburg: Hoffmann und Campe.
Riedel, U. (2005). „Alle Macht den Räten?“ Politikberatung durch Ethikgremien in der Biopolitik. In: Düwell M. \& Neumann (Hrsg.). Wieviel Ethik verträgt die Medizin? Paderborn: Mentis, S. 367-376.

Schicktanz S. \& Schweda M. (2007). Zwischen Expertokratie und Bürgerbeteiligung. Wer sollte bei ethischer Politikberatung eine Rolle spielen? In: Gen-ethischer Informationsdienst, 23. Jg., Nr. 182 (Juni), S. 57-60.

Schönberger, R. (Hrsg.) (2001). Thomas von Aquin. Über sittliches Handeln. Summa theologiae I-II q. 18-21. Stuttgart: Reclam.

Spaemann, R. (2001). Einleitung. In: Thomas von Aquin (2001). Über sittliches Handeln. Summa theologiae I-II q. 18-21. Lateinisch/Deutsch. Übersetzt, kommentiert und herausgegeben von Rolf Schönberger. Einleitung von Robert Spaemann. Stuttgart: Reclam, S. 7-18.

Thomas von Aquin (2001). Über sittliches Handeln. Summa theologiae I-II q. 18-21. Lateinisch/Deutsch. Übersetzt, kommentiert und herausgegeben von Rolf Schönberger. Einleitung von Robert Spaemann. Stuttgart: Reclam.

van den Daele, W. (2001). Von moralischer Kommunikation zur Kommunikation über Moral. Reflexive Distanz in diskursiven Verfahren. In: Zeitschrift für Soziologie, 30. Jg., Heft 1 (Februar), S. 4-22.

van den Daele, W. (2008). Streitkultur. Über den Umgang mit unlösbaren moralischen Konflikten im Nationalen Ethikrat. In: Gosewinkel D. \& Schuppert G. F. (Hrsg.). Politische Kultur im Wandel von Staatlichkeit (WZB-Jahrbuch 2007). Berlin: edition sigma, S. 357-384.

Vöneky, S. (2009). Ethische Experten und moralischer Autoritarismus. In: Vöneky S. \& Hagedorn C. \& Clados, M. \& von Achenbach, J. (Hrsg.). Legitimation ethischer Entscheidungen im Recht. Interdisziplinäre Untersuchungen. Berlin/Heidelberg/New York: Springer, S. 85-98.

Weber M. (1988). Politik als Beruf. In: Gesammelte Politische Schriften, 5. Auflage, Tübingen: Mohr Siebeck, S. 505-560.

Weilert, A. K. (2011). Thesen zu „Ethischer Politikberatung“. In: Zeitschrift für Politikberatung, 4. Jg., Heft 1, S. 39-41.

Weilert, A. K. \& Hildmann, P. W. (Hrsg.). (2012). Ethische Politikberatung. Baden-Baden: Nomos Verlagsgesellschaft.

Zotti, S. (2009). Ethische Politikberatung. Anmerkungen zur Frage der Legitimation von Expertenkommissionen im bioethischen Diskurs. In: Vöneky S. \& Hagedorn C. \& Clados, M. \& von Achenbach, J. (Hrsg.). Legitimation ethischer Entscheidungen im Recht. Interdisziplinäre Untersuchungen. Berlin/Heidelberg/New York: Springer, S. 99-114.

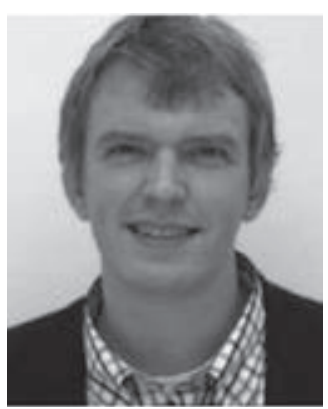

Prof. Dr. Axel Bohmeyer, ist Professor für Erziehungswissenschaft mit den Schwerpunkten Erziehungsphilosophie, Bildung und Erziehung, Pädagogische Anthropologie sowie Bildung und Partizipation an der Katholischen Hochschule Berlin. Er ist Geschäftsführer des Instituts für Christliche Ethik und Politik. E-Mail: Axel.Bohmeyer@KHSB-Berlin.de.

16 Doch auch am irrenden Gewissen könnte sich ein moralischer Streit entzünden. Wie lässt sich mit den Moralisten verfahren, die die moralische Redlichkeit der Berufung auf die Autorität des irrenden Gewissens bestreiten? Dann gilt: Ethische Politikberater sollten vor solchen Personen warnen! 